
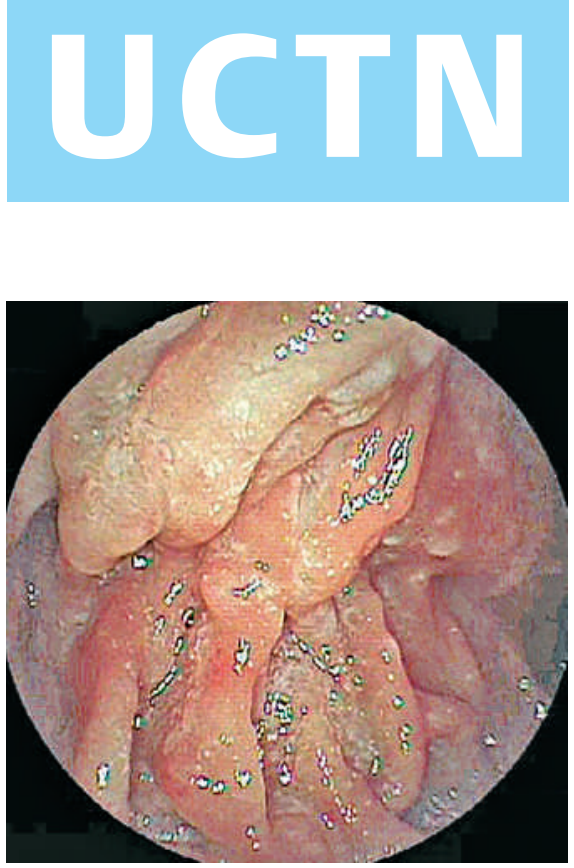

Figure 1 Esophagogastroduodenoscopy identified a whitish granular elevation in the descending duodenum in patient 1.

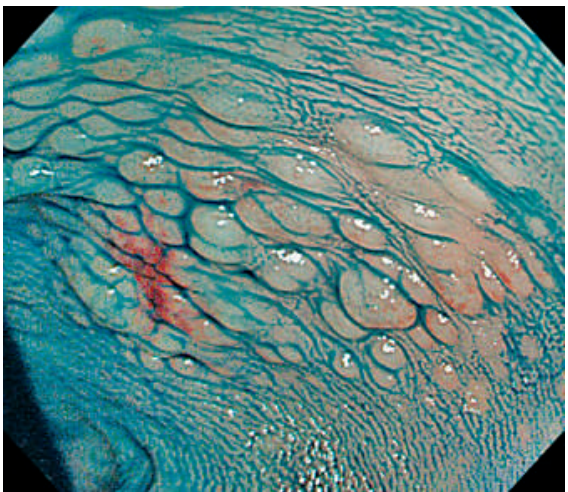

Figure 2 Colonoscopy in patient 2 revealed a flat protrusion in the rectum.

Two patients with follicular lymphoma of the gastrointestinal tract were referred to our institution for capsule endoscopy. The first patient, a man aged 64 years, had undergone esophagogastroduodenoscopy, which had shown a whitish, granular, elevated area in the descending duodenum (Figure 1). The second patient, a $60-$ year-old woman, had a flat protrusion in the rectum (Figure 2 ). In both cases, endoscopic biopsy of these lesions revealed follicular lymphoma (Figure 3).

In patient 1 , capsule endoscopy images showed patchy whitish nodules in the proximal and middle parts of the small intestine (Figure 4) [1]. The capsule study also identified thickened mucosal folds with ulceration in the proximal part of the small intestine. However, no apparent

\title{
Capsule endoscopy findings in intestinal follicular lymphoma
}

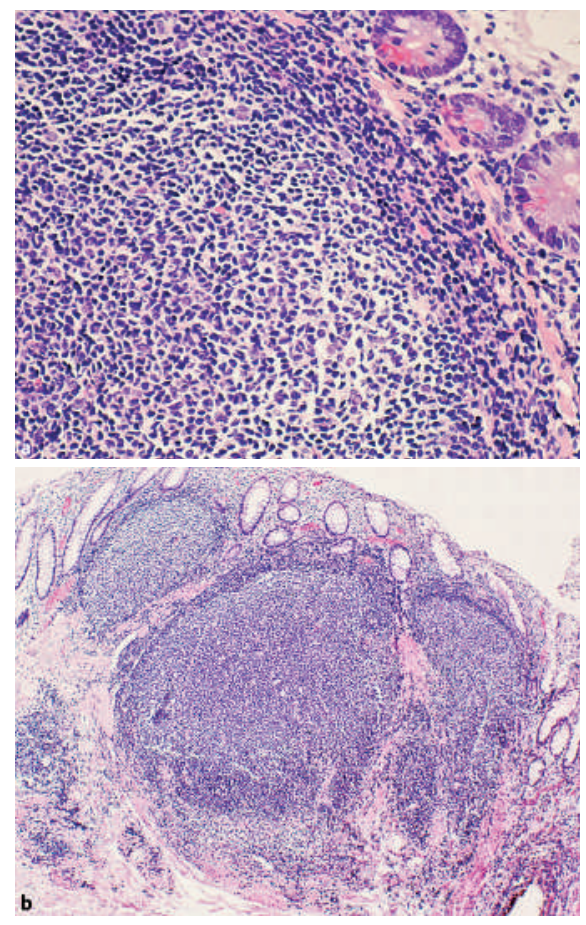

Figure 3 Endoscopic biopsies were taken from the duodenal lesion in patient 1 and from the rectal elevation in patient 2 . Histological views of the endoscopic biopsy specimens after staining with hematoxylin and eosin showed proliferation of small- to medium-sized atypical lymphoid cells (magnification $\times 200$, a) in a follicular pattern (magnification $\times 100, \mathbf{b})$.

abnormality was found in the distal part of the small intestine. Based on the capsule endoscopic and computed tomographic findings, the clinical stage was assessed as stage II [2]. We treated the patient with rituximab, cyclophosphamide, doxorubicin, vincristine, and predonisolone (R-CHOP) chemotherapy. Repeat capsule endoscopy after six cycles of R-CHOP therapy showed an obvious improvement in the intestinal disease. In patient 2, capsule endoscopy detected diminutive whitish granules and white villi in the proximal and middle parts of the small intestine (Figure 5), but, again, no mucosal lesions in the distal small intestine. Because her gastrointestinal lymphoma was assessed as being stage I [2], and because it was not causing any clinical symptoms, the patient was not given

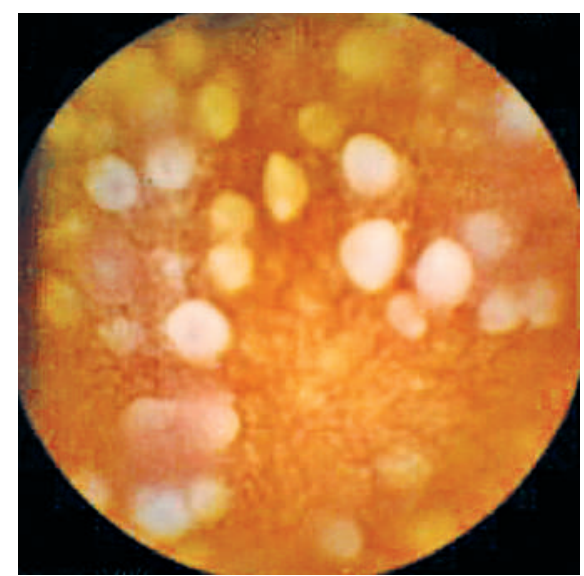

Figure 4 Capsule endoscopic image showing multiple whitish nodules in the proximal part of the small intestine in patient 1.

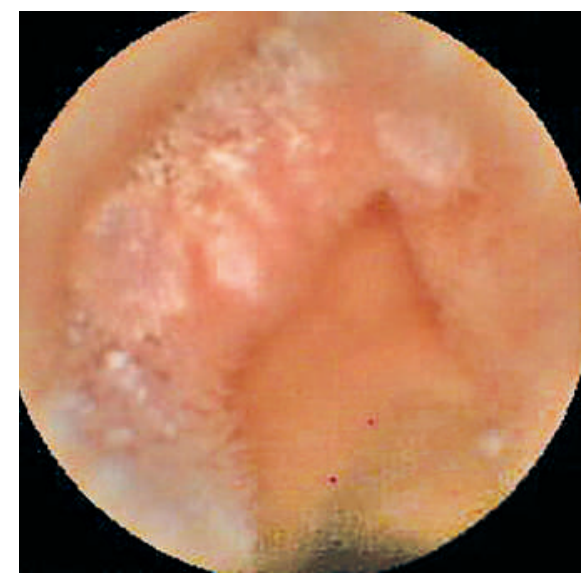

Figure 5 Capsule endoscopy revealed diminutive, whitish granules and white villi in the proximal part of the small intestine in patient 2.

any aggressive treatment but has been kept under careful observation. The lymphoma has not progressed during the subsequent period of 2 years.

Follicular lymphoma, which accounts for $1 \%-3 \%$ of primary gastrointestinal lymphomas [3], is believed to involve the terminal ileum and to develop into obstructing intramural masses [4]. Our cases suggest that whitish nodules and granules in the upper part of the small intestine are characteristic findings that

DOI: 10.1055/s-2006-945152 
help differentiate follicular lymphoma from other histologic types. Capsule endoscopy could therefore become an essential procedure for the diagnosis of follicular lymphoma of the gastrointestinal tract.

Endoscopy_UCTN_Code_CCL_1AC_2AC

M. Esaki ${ }^{1}$, T. Matsumoto ${ }^{1}$, S. Nakamura', H. Suekane' ${ }^{2}$ Y. Ohjï ${ }^{3}$, T. Yao ${ }^{3}$, M. Iida ${ }^{1}$

${ }^{1}$ Department of Medicine and Clinical Science, Graduate School of Medical Sciences, Kyushu University, Fukuoka, Japan

${ }^{2}$ Division of Gastroenterology, Yamaguchi Red Cross Hospital, Yamaguchi, Japan

${ }^{3}$ Department of Anatomic Pathology, Graduate School of Medical Sciences, Kyushu University, Fukuoka, Japan.
References

${ }^{1}$ Korman LY, Delvaux M, Gay G et al. Capsule endoscopy structured terminology (CEST): proposal of a standardized and structured terminology for reporting capsule endoscopy procedures. Endoscopy 2005; 37: $951-$ 959

${ }^{2}$ Zucca E, Roggero E, Bertoni F et al. Primary extranodal non-Hodgkin's lymphomas. Part 1: gastrointestinal, cutaneous and genitourinary lymphomas. Ann Oncol 1997; 8: 727 737

${ }^{3}$ Shia J, Teruya-Feldstein J, Pan D et al. Primary follicular lymphoma of the gastrointestinal tract: a clinical and pathologic study of 26 cases. Am J Surg Pathol 2002; 26: 216 224

${ }^{4}$ LeBrun DP, Kamel OW, Cleary ML et al. Follicular lymphomas of the gastrointestinal tract: pathologic features in 31 cases and bcl-2 oncogenic protein expression. Am J Pathol 1992; 140: 1327-1335
Corresponding author

\section{Esaki, MD}

Department of Medicine and Clinical Science

Graduate School of Medical Sciences Kyushu University

Maidashi 3-1-1

Higashi-ku

Fukuoka 812-8582

Japan

Fax: $\quad$ +81-92-642-5273

Email: mesaki@intmed2.

med.kyushu-u.ac.jp 